\title{
Acute bilateral vision deficit as the initial symptom in Guillain-Barre syndrome: A case report
}

\author{
HUI-JUN WEN \\ Department of Neurology, Baoji Municipal Central Hospital, Baoji, Shaanxi 721008, P.R. China
}

Received September 27, 2017; Accepted January 8, 2018

DOI: $10.3892 /$ etm.2018.6465

\begin{abstract}
Guillain-Barre syndrome (GBS) is a rare autoimmune-mediated peripheral neuropathy with an acute onset. GBS is associated with posterior cranial nerve injury including the facial, oculomotor, glossopharyngeal and vagus nerves. A 43-year-old female presented with worsening blurred binocular vision accompanied by diplopia, bilateral ptosis, speech difficulties, dysphagia, extremity numbness and weakness of the limbs. A neurological examination was conducted along with magnetic resonance imaging of the brain and spinal cord, electromyography, cerebrospinal fluid analysis and visual evoked potential tests, which led to a diagnosis of GBS. Following immunoglobulin administration, the patient's neurological deficiencies were markedly relieved and they were discharged following a 3-week observation period. To the best of our knowledge, this is the first reported case of GBS associated with cranial nerve damage presenting with bilateral visual deterioration as the initial symptom. It is necessary to emphasize that early diagnosis and commencing the immunological treatment in a timely manner resulted in a favorable prognosis for the patient.
\end{abstract}

\section{Introduction}

Guillain-Barre Syndrome (GBS) is a rare immune-mediated acute inflammatory demyelinating polyneuropathy with a rapid onset (1). The annual incidence rate of GBS ranges from 0.81-1.89 per 100,000 people (2) and males are more frequently affected than females (3). Previous studies investigating GBS have focused on assessing objective parameters, including

Correspondence to: Dr Hui-Jun Wen, Department of Neurology, Baoji Municipal Central Hospital, 8 Jiangtan Road, Baoji, Shaanxi 721008, P.R. China

E-mail: whjsq2008@163.com

Abbreviations: GBS, Guillain-Barre syndrome; IVIG, intravenous immunoglobulin; MRI, magnetic resonance imaging; VEP, visual evoked potential

Key words: autoimmune polyneuropathy, Guillain-Barre syndrome, multiple cranial nerve damage, visual deterioration medical history, physical, laboratory and electrophysiological examinations (4).

Clinical features of GBS include areflexia and limb weakness, with additional sensory loss being reported in certain cases (5). Symptoms can further develop into neuromuscular paralysis, implicating bulbar, facial and respiratory function with maximum severity in the initial 2-4 weeks of onset (5). Multiple cranial neuropathies are a rare variant of GBS, accounting for $5 \%$ of patients (6). Owing to the rarity of features, this GBS variant is not often considered in the differential diagnosis due to its lack of progression to limb weakness (7). To date, there are few studies that describe pure cranial neuropathy with areflexia and normal motor and sensory functions as a rare GBA subtype $(7,8)$. Previous reports also described GBS presented with cranial nerve damage as the initial symptom (9-11). Demyelination is often an initial sign of GBS with subsequent damage to the facial, oculomotor, glossopharyngeal and vagus nerves. Although the clinical course, severity and prognosis of the different GBS subtypes are variable, intravenous immunoglobulin (IVIG) and plasma exchange are proven to be effective treatments (5). To the best of our knowledge, the current study describes the first reported case of GBS with cranial nerve impairment, in which visual impairment was the initial symptom.

\section{Case report}

The present case report was approved by the Ethics Committee of Baoji Municipal Central Hospital (Baoji, China) and the patient provided written informed consent.

A 43-year-old female presented with binocular vision loss for five days and was admitted to the Department of Neurology, Baoji Municipal Central Hospital on December 20th 2016 for potential cranial neuropathy. During the initial physical examination, deterioration of near and distant vision was identified. The patient recently ( 2 weeks prior) recovered from an upper respiratory tract infection and the rest of, her medical history was otherwise unremarkable. The following neurological exams were normal: Limb muscular tension (grade 5), response to sensory stimuli, synesthesia, reflexes of the bilateral biceps, triceps, knees and Achilles tendons and bilateral Babinski (data not shown) (12). Those investigations followed the standard protocols of neurological investigation. On day 3 post-admission, the patient's symptoms intensified, with worsening of the blurred binocular vision accompanied by diplopia, 
bilateral ptosis, speech difficulty, excessive coughing during drinking, dysphagia, extremity numbness and weakness of the limbs (data not shown).

A neurological examination revealed bilateral loss of visual acuity, with 20/30 for the right eye and 20/25 for the left (data not shown). The following were also abnormal: Weakened limb muscular tension (grade 3 ) and limb reflexes, as well as weakened sensory feeling upon the direct physical examination (data not shown). However, the deep sensory reflexes and bilateral Babinski signs were normal (data not shown). Optical coherence tomography revealed that the peripapillary retinal nerve fiber layer was thickened on each side, suggesting bilateral edema of the optic nerves (data not shown).

Cerebral magnetic resonance imaging (MRI) and cervical spinal MRI were normal (Fig. 1). The patient rejected a MRI of the optic nerves due to the financial burden. Visual evoked potential (VEP) revealed that the latency of the bilateral full-field P100 was prolonged and the amplitude was low (Fig. 2). Electromyography revealed severe impairment of the sensory and motor fibers in the bilateral median, ulnar, tibial and common peroneal nerves (Tables I and II).

Next, a lumbar puncture was performed to collect the cerebrospinal fluid (CSF) 5 days post-admission as previously described (13). CSF analysis revealed albuminocytological dissociation and protein-cell separation (data not shown). The white blood cell count was $0.009 \times 10^{9}$ cells $/ 1$ (normal value, $0-0.008 \times 10^{9}$ cells $/ 1$ ) and the red blood cell count was 0 (normal value, 0-2 individual/HP). Protein, chlorine and sugar concentrations were $862 \mathrm{mg} / 1$ (normal value, 0.15-0.45 g/l), $129.6 \mathrm{mmol} / \mathrm{l}$ (normal value, $120-130 \mathrm{mmo} / \mathrm{l}$ ) and sugar $3.69 \mathrm{mmol} / 1$ (normal value, 2.5-4.4 mmo/1), respectively. Analysis of aquaporin-4 immunoglobulin G (AQP4-IgG) in the peripheral blood and CSF were normal (data not shown). The peripheral blood anti-Sm antibody, anti-cyclic citrullinated peptide (anti-CCP) antibodies, rheumatoid factor and neutrophil cytoplasmic autoantibodies were all normal (data not shown).

Analyses of the clinical symptoms, neurological examination, electromyography, brain and spinal cord MRI, and peripheral blood and CSF findings led to a diagnosis of GBS. Following the identification of GBS, the patient immediately received IVIG treatment $(0.4 \mathrm{~g} / \mathrm{kg} / \mathrm{day})$. A marked improvement in the patient's blurred vision, diplopia, ptosis, speech, dysphagia and limb activities were observed 5 days later. The patient regained the ability to hold objects in their hands and walk independently. Additionally, dexamethasone immune modulating therapy (10 mg/day, once daily) was administrated for 14 days and a neurotrophic treatment (mecobalamin; 1,000 $\mu \mathrm{g}$, once daily) was administered for 21 days.

The patient was discharged following 3 weeks when their blurred vision had returned to normal and with a marked improvement in her neurological functions. Specifically, the initially observed diplopia, bilateral ptosis, speech difficulty, excessive coughing during drinking, dysphagia, extremity numbness and limb weakness were improved but not completely restored to normal. Additionally, the VEP revealed that the latency and the amplitude of the bilateral full-field P100, N75 and N145 had returned to normal levels (Fig. 3) and the patient was able to live independently.
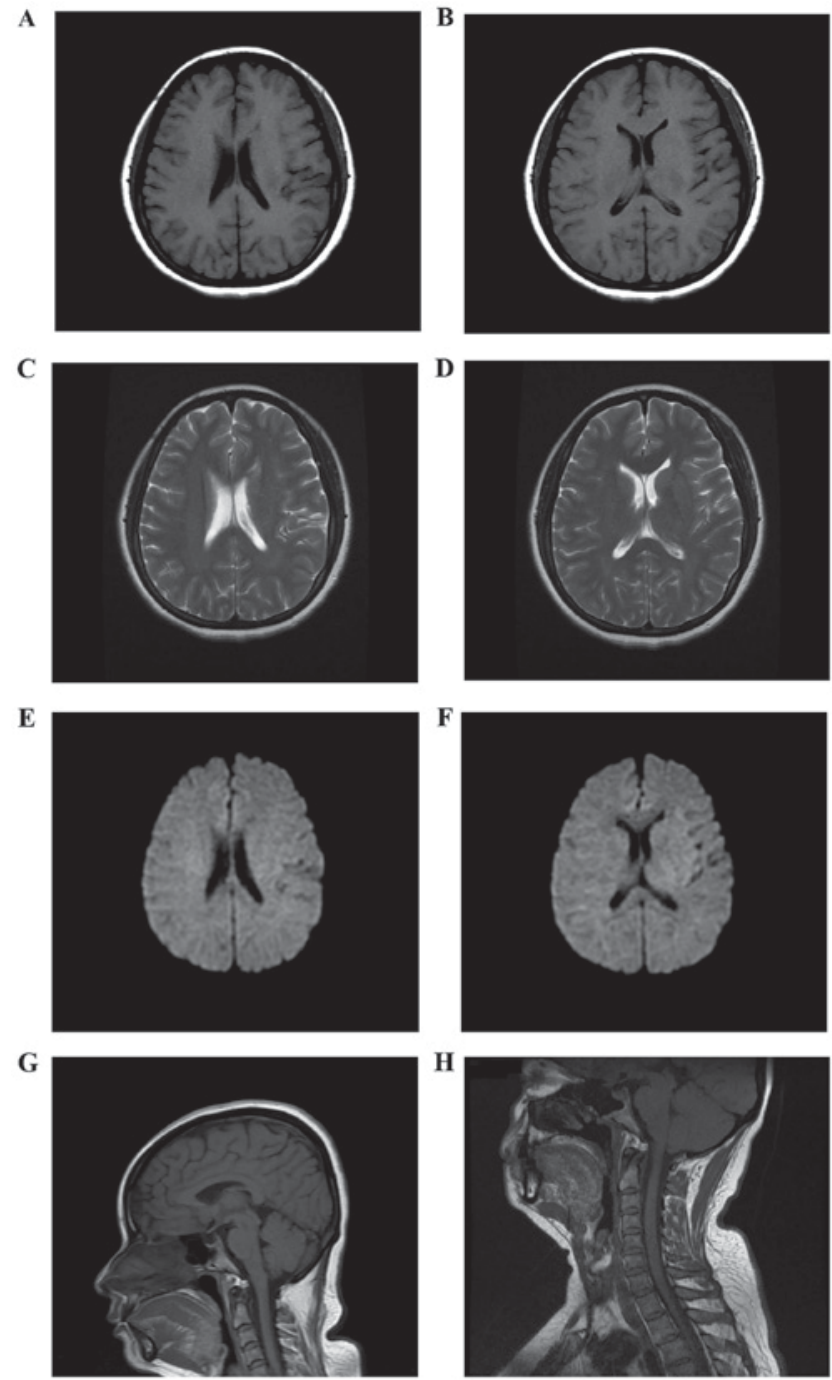

Figure 1. Cerebral cervical spine MRI of a 43-year-old female with Guillain-Barre syndrome presenting with acute bilateral vision deficit. (A-F) Brain MRI indicated bilateral hemispheric symmetry and no focal abnormalities of signal. The ventricles of the brain, midline structures, subtentorial cerebellar and brainstem appeared normal. (G) A sagittal scan revealed normal shape and size of the brainstem and pituitary. $(\mathrm{H})$ Cervical MRI indicated normal cervical sequence. The physiological curvature of the cervical spine appeared normal, the spinal cord had no abnormal changes and no stenosis. MRI, magnetic resonance imaging.

\section{Discussion}

GBS is an autoimmune disease caused by peripheral nerve demyelination. In the present study, a rare case of GBS was reported in which the patient presented with cranial nerve damage and visual deterioration as the initial symptom.

In 1966, Morley and Reynolds (14) drew attention to the possibility of optic neuritis associated with GBS. In 2015, a 10-year-old child with GBS developed bilateral optic neuritis during the later stages of the illness (15). Similarly, a 14-year-old male with GBS presented with hyperreflexia and bilateral papillitis (16). In the present case, the initial symptoms of GBS were binocular visual deterioration followed by the development of diplopia and ptosis, which indicated a lesion involving the bilateral oculomotor nerve. Subsequently, the patient's inarticulacy and swallowing difficulties were 
Table I. Conduction velocity of the sensory nerves.

\begin{tabular}{|c|c|c|c|c|c|c|c|}
\hline \multirow[b]{2}{*}{ Limb sensory nerve conduction } & \multirow[b]{2}{*}{$\mathrm{L} / \mathrm{R}$} & \multirow[b]{2}{*}{$\mathrm{LAT}(\mathrm{m} / \mathrm{sec})$} & \multirow[b]{2}{*}{$\operatorname{AMP}(\mu \mathrm{v})$} & \multirow[b]{2}{*}{$\mathrm{DN}(\mathrm{cm})$} & \multirow[b]{2}{*}{$\mathrm{NCV}(\mathrm{m} / \mathrm{sec})$} & \multicolumn{2}{|c|}{ Normal reference values } \\
\hline & & & & & & $\operatorname{AMP}(\mu \mathrm{v})$ & $\mathrm{NCV}(\mathrm{m} / \mathrm{sec})$ \\
\hline \multirow[t]{2}{*}{ Median } & $\mathrm{L}$ & 3.17 & 11.20 & 11.60 & 36.90 & 12.60 & 42.10 \\
\hline & $\mathrm{R}$ & 3.18 & 11.30 & 11.00 & 37.60 & 12.50 & 41.70 \\
\hline \multirow[t]{2}{*}{ Ulnar } & $\mathrm{L}$ & 3.28 & 5.40 & 12.00 & 42.90 & 6.70 & 44.80 \\
\hline & $\mathrm{R}$ & 3.20 & 4.50 & 11.00 & 42.60 & 6.60 & 43.90 \\
\hline \multirow[t]{2}{*}{ Tibial } & $\mathrm{L}$ & 8.40 & 0.16 & 17.50 & 20.30 & 0.42 & 35.60 \\
\hline & $\mathrm{R}$ & 6.50 & 0.25 & 16.00 & 25.40 & 0.30 & 34.60 \\
\hline \multirow[t]{2}{*}{ Common peroneal } & $\mathrm{L}$ & 7.19 & 0.24 & 26.00 & 36.90 & 0.60 & 46.39 \\
\hline & $\mathrm{R}$ & 7.80 & 0.21 & 27.00 & 33.80 & 0.60 & 46.53 \\
\hline
\end{tabular}

L/R, left/right; AMP, amplitude; DN, distance; LAT, latency; NCV, nerve conduction velocity.

Table II. Conduction velocity of the motor nerves.

\begin{tabular}{lcccc}
\hline Limb motor nerve conduction & L/R & LAT (m/sec) & AMP (mv) & Normal AMP values (mv) \\
\hline Median & L & 4.39 & 5.10 & 7.00 \\
& R & 4.73 & 5.80 & 7.00 \\
Ulnar & $\mathrm{L}$ & 3.62 & 5.50 & 7.00 \\
& $\mathrm{R}$ & 3.47 & 5.00 & 7.00 \\
Tibial & $\mathrm{L}$ & 6.21 & 2.40 & 4.00 \\
Common peroneal & $\mathrm{R}$ & 6.32 & 2.30 & 4.00 \\
& $\mathrm{~L}$ & 5.59 & 2.10 & 4.00 \\
& $\mathrm{R}$ & 4.41 & 2.70 & 4.00 \\
\hline
\end{tabular}

L/R, left/right; AMP, amplitude; LAT, latency.

indicative of a lesion involving the glossopharyngeal and vagus nerves. Extremity numbness and weakness of the limbs indicated a lesion involving the peripheral nerves.

Following 5 days of IVIG therapy, the above clinical symptoms markedly improved. This was attributed to the ability of IVIG treatment to adjust humoral immunity and inhibit the production of autoantibodies, thereby reducing complement-mediated impairment (17). In addition, the patient demonstrated improved visual acuity, diplopia and ptosis, which suggests the therapeutic efficacy of IVIG treatment in GBS cases involving cranial nerve damage consistent with previously published data (18).

Atypical cases of GBS may be easily misdiagnosed, therefore, I recommend that suspected GBS should be confirmed by the collective analysis of clinical symptoms, neurological examination, electromyography, brain and spinal cord MRI and CSF examination. These examinations should provide an accurate diagnosis that distinguishes GBS from other intracranial diseases, including brain stem encephalitis, pharyngeal cervical arm powerlessness and acute ophthalmoplegia $(7,19)$. Similarly, it is necessary to exclude systemic lupus erythematosus (20), rheumatoid arthritis (21), antiphospholipid antibody syndrome (22) and neuromyelitis optica (23), which may also potentially cause nervous system damage.
In the present study, the patient's symptoms progressed rapidly, which eliminated the possibility of nervous system damage due to rheumatoid arthritis. In addition, the patient was previously healthy and had no clinical manifestations, including body rash, kidney damage, joint pain in the extremities, pulmonary infection or heart disease. The level of anti-Sm antibody was normal, which excludes nervous system damage caused by systemic lupus erythematosus. Additionally, the patient exhibited no symptoms of headache, vomiting, limb twitching or disturbance of consciousness and results of a routine blood examination, anti-cardiolipin antibodies and anti-neutrophil cytoplasmic antibodies in the serum were normal. The cerebral and cervical MRI revealed no abnormal signals, which excluded possible nervous system damage caused by anti-phospholipid antibody syndrome or granulomatous angiitis. Although the patient presented with vision loss and signs of optic nerve damage, the absence of sphincter disturbance and normal findings in the cervical MRI, as well as the AQP4-IgG and CSF, led to the elimination of neuromyelitis optica from the differential diagnosis.

In conclusion, the present study reports a rare case of GBS associated with cranial nerve damage where the initial presenting symptom was sudden deterioration of binocular vision. I hypothesize that the patient developed GBS 


\begin{tabular}{|c|c|c|c|c|}
\hline Protocol / Run & $\begin{array}{l}\text { N75 } \\
\text { ms }\end{array}$ & $\begin{array}{c}\text { P100 } \\
\text { ms }\end{array}$ & $\begin{array}{c}\mathrm{N} 145 \\
\mathrm{~ms}\end{array}$ & $\begin{array}{c}\mathrm{P} 100 \\
\mu \mathrm{V}\end{array}$ \\
\hline \multicolumn{5}{|l|}{ L - VEP } \\
\hline $1.1 \mathrm{O1}-\mathrm{Fz}$ & 74.10 & 114.60 & 160.50 & 0.06 \\
\hline $1.2 \mathrm{Oz}-\mathrm{Fz}$ & 85.50 & 116.10 & 169.20 & 0.78 \\
\hline $1.3 \mathrm{O} 2-\mathrm{Fz}$ & 76.50 & 122.40 & 167.10 & 1.40 \\
\hline \multicolumn{5}{|l|}{ R - VEP } \\
\hline $1.1 \mathrm{O}-\mathrm{Fz}$ & 115.50 & 138.00 & 160.50 & 1.40 \\
\hline $1.2 \mathrm{Oz}-\mathrm{Fz}$ & 117.90 & 147.30 & 161.10 & 1.80 \\
\hline $1.3 \mathrm{O} 2-\mathrm{Fz}$ & 117.90 & 149.10 & 171.90 & 1.70 \\
\hline
\end{tabular}

B

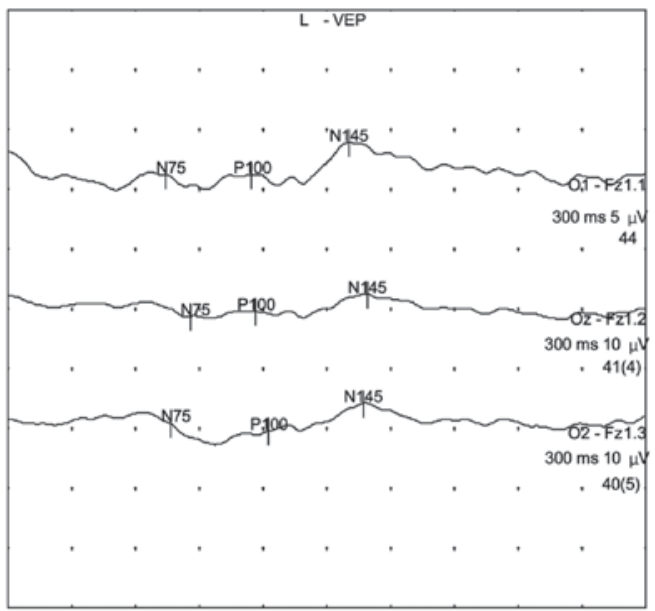

C

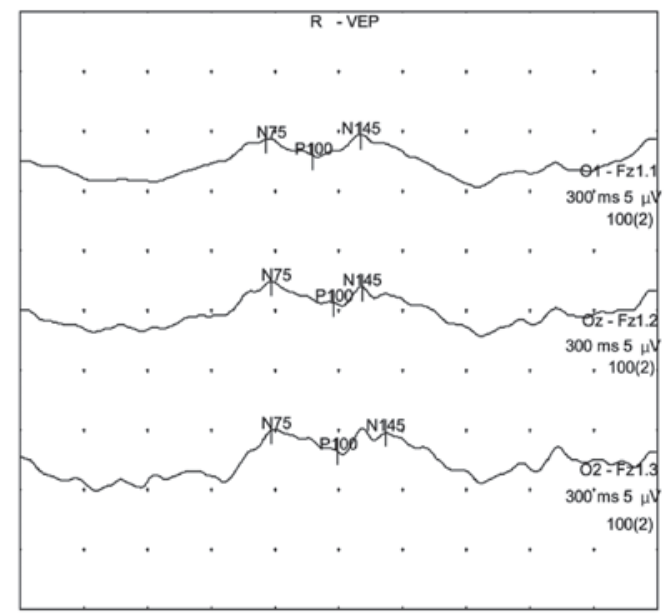

Figure 2. Bilateral VEP of a 43-year-old female affected by Guillain-Barre syndrome prior to treatment. (A) Tabular numerical indicated impaired binocular vision with prolonged latency of the bilateral full-field and decreased amplitude. (B) The pattern reversal of VEP for the left eye. N75 waves demonstrated normal latency while the P100 and N145 waves had prolonged latency. (C) The pattern reversal of VEP for the right eye. P100, N145 and N75 waves from the right eye displayed prolonged latency and poor differentiation. VEP, visual evoked potential.

subsequent to a recent upper respiratory tract infection. The diagnosis of GBS was based on clinical symptoms, neurological examinations, electromyography, brain and spinal cord MRI and CSF examination. In addition, the curative efficacy of IVIG therapy in GBS treatment was demonstrated. IVIG has multiple roles in the immune system, including the provision of anti-idiotypic antibodies, blocking macrophage

\begin{tabular}{|c|c|c|c|c|}
\hline Protocol / Run & $\begin{array}{c}\text { N75 } \\
\text { ms }\end{array}$ & $\begin{array}{l}\text { P100 } \\
\text { ms }\end{array}$ & $\begin{array}{c}\text { N145 } \\
\text { ms }\end{array}$ & $\begin{array}{c}P 100 \\
\mu V\end{array}$ \\
\hline \multicolumn{5}{|l|}{ L - VEP } \\
\hline $1.1 \mathrm{O}-\mathrm{Fz}$ & 74.40 & 108.30 & 153.90 & 5.10 \\
\hline $1.2 \mathrm{Oz}-\mathrm{Fz}$ & 71.40 & 109.20 & 153.00 & 6.30 \\
\hline $1.3 \mathrm{O} 2-\mathrm{Fz}$ & 70.80 & 110.10 & 153.00 & 3.40 \\
\hline \multicolumn{5}{|l|}{$R$ - VEP } \\
\hline $1.1 \mathrm{O} 1-\mathrm{Fz}$ & 71.40 & 110.40 & 153.00 & 3.00 \\
\hline $1.2 \mathrm{Oz}-\mathrm{Fz}$ & 74.10 & 113.70 & 149.40 & 6.20 \\
\hline $1.3 \mathrm{O} 2-\mathrm{Fz}$ & 73.50 & 113.10 & 151.20 & 0.34 \\
\hline
\end{tabular}

B
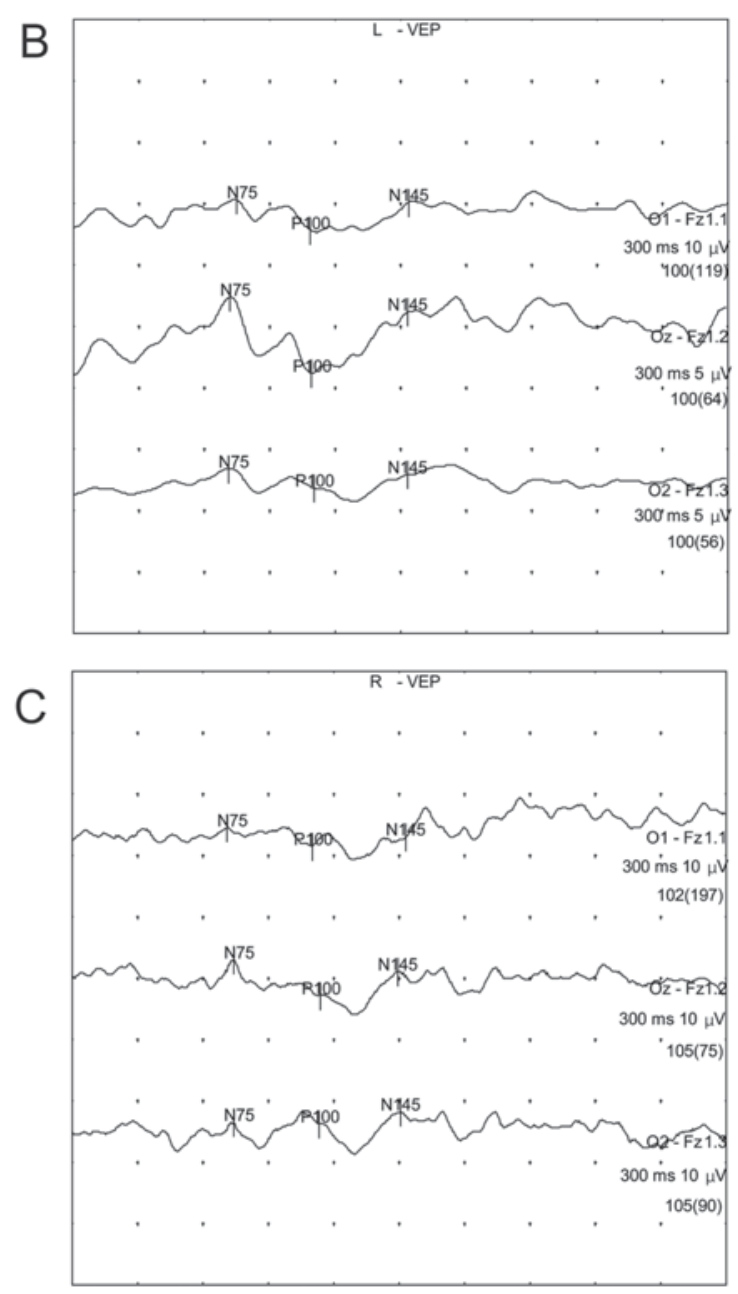

Figure 3. Bilateral VEP of a 43-year-old female with Guillain-Barre syndrome following treatment. (A) Tabular numerical indicated restored binocular vision with normal latency of the bilateral full-field and normal amplitude. (B) The pattern reversal of VEP for the left eye. N75, P100 and N145 waves demonstrated normal latency and amplitude. (C) N75, P100 and N145 waves from the right eye displayed normal latency and amplitude. VEP, visual evoked potential.

Fc receptors, inhibiting complement activity and modulating central B- and T-cell function (24). Due to the autoimmune nature of GBS, immunoglobulin therapy has been proposed as a potentially effective treatment (18). Nevertheless, to the best of my knowledge, the addition of immune-modulatory agents (dexamethasone) and neurotrophic treatment (mecobalamin) to IVIG treatment enhanced the therapeutic efficiency. 


\section{Acknowledgements}

The author would like to thank Dr Jun-Wen Wang, Department of Neurology, Baoji Municipal Central Hospital, 8 Jiangtan Road, Baoji, Shaanxi 721008, P.R. China for his technical assistance in performing the present study.

\section{Funding}

No funding was received.

\section{Availability of data and materials}

The analyzed data sets generated during the present study are available from the corresponding author on reasonable request.

\section{Author contribution}

Hui-Jun Wen conceived, designed and drafted the manuscript, was responsible for the acquisition analysis, revision and interpretation of data and approved of the final version to be published. Hui-Jun Wen agrees to be accountable for all aspects of the work in ensuring that questions related to the accuracy or integrity of any part of the work.

\section{Ethics approval and consent to participate}

The present case report was approved by the Ethics Committee of Baoji Municipal Central Hospital (Baoji, China) and the patient provided written informed consent.

\section{Patient consent for publication}

Written informed consent was obtained for publication.

\section{Competing interests}

The author declares that he has no competing interests.

\section{References}

1. Thomas NH: Diagnosis and management of Guillain-Barre syndrome. Curr Paediatr 15: 287-291, 2005.

2. Sejvar JJ, Baughman AL, Wise M and Morgan OW: Population incidence of Guillain-Barré syndrome: A systematic review and meta-analysis. Neuroepidemiology 36: 123-133, 2011.

3. Bogliun G and Beghi E; Italian GBS Registry Study Group: Incidence and clinical features of acute inflammatory polyradiculoneuropathy in Lombardy, Italy, 1996. Acta Neurol Scand 110: 100-106, 2004.
4. Fokke C, van den Berg B, Drenthen J, Walgaard C, van Doorn PA and Jacobs BC: Diagnosis of Guillain-Barré syndrome and validation of Brighton criteria. Brain 137: 33-43, 2014.

5. van Doorn PA, Ruts L and Jacobs BC: Clinical features, pathogenesis, and treatment of Guillain-Barré syndrome. Lancet Neurol 7: 939-950, 2008.

6. Lin JJ, Hsia SH, Wang HS, Lyu RK, Chou ML, Hung PC, Hsieh MY and Lin KL: Clinical variants of Guillain-Barré syndrome in children. Pediatr Neurol 47: 91-96, 2012.

7. Yu JY, Jung HY, Kim CH, Kim HS and Kim MO: Multiple cranial neuropathies without limb involvements: Guillain-Barre syndrome variant. Ann Rehabil Med 37: 740-744, 2013.

8. Lyu RK and Chen ST: Acute multiple cranial neuropathy. A variant of Guillain-Barré syndrome. Muscle Nerve 30: 433-436, 2004.

9. Sudulagunta SR, Sodalagunta MB, Sepehrar M, Khorram H, Bangalore Raja SK, Kothandapani S, Noroozpour Z, Aheta Sham M, Prasad N, Sunny SP, et al: Guillain-Barré syndrome: Clinical profile and management. Ger Med Sci 13: Doc16, 2015.

10. van den Berg B, Walgaard C, Drenthen J, Fokke C, Jacobs BC and van Doorn PA: Guillain-Barré syndrome: Pathogenesis, diagnosis, treatment and prognosis. Nat Rev Neurol 10: 469-482, 2010.

11. Rafii MS: Case 14: A woman with bilateral Bell's palsy. Med Gen Med 8: 23, 2006.

12. Bogousslavsky J and Fisher M: Textbook of neurology. Butterworth-Heinemann Co: 3-18, 1998.

13. Peskind ER, Riekse R, Quinn JF, Kaye J, Clark CM, Farlow MR, Decarli C, Chabal C, Vavrek D, Raskind MA and Galasko D: Safety and acceptability of the research lumbar puncture. Alzheimer Dis Assoc Disord 19: 220-225, 2005.

14. Morley JB and Reynolds EH: Papilloedema and the Landry-Guillain-Barré syndrome. Case reports and a review. Brain 89: 205-222, 1966.

15. Deshmukh IS, Bang AB, Jain MA and Vilhekar KY: Concurrent acute disseminated encephalomyelitis and Guillain-Barre syndrome in a child. J Pediatr. Neurosci 10: 61-63, 2015.

16. Incecik F, Herguner OM, Besen S, Yar K and Altunbasak S: Guillain-Barré syndrome with hyperreflexia and bilateral papillitis in a child. J Pediatr Neurosci 11: 71-73, 2016.

17. Hughes RA, Swan AV and van Doorn PA: Intravenous immunoglobulin for Guillain-Barré syndrome. Cochrane Database Syst Rev: CD002063, 2014.

18. Raphaël JC, Chevret S, Hughes RA and Annane D: Plasma exchange for Guillain-Barré syndrome. Cochrane Database Syst Rev: CD001798, 2012.

19. Edvardsson B and Persson S: Polyneuritis cranialis presenting with anti-GQ1b IgG antibody. J Neurol Sci 281: 125-126, 2009.

20. Ali N, Rampure R, Malik F, Jafri SI and Amberker D: Guillain-Barré syndrome occurring synchronously with systemic lupus erythematosus as initial manifestation treated successfully with low-dose cyclophosphamide. J Community Hosp Intern Med Perspect 6: 30689, 2016.

21. Silburn S, McIvor E, McEntegart A and Wilson H: Guillain-Barré syndrome in a patient receiving anti-tumour necrosis factor alpha for rheumatoid arthritis: A case report and discussion of literature. Ann Rheum Dis 67: 575-576, 2008.

22. Hara M: Intravenous immunoglobulin (IVIG). Nihon Rinsho 67: 599-605, 2009 (In Japanese).

23. Kusunoki S: Neuroimmunological diseases: Update. Nihon Rinsho 71: 771-777, 2013 (In Japanese).

24. Hughes RA: Intravenous immunoglobulin for chronic inflammatory demyelinating polyradiculoneuropathy: The ICE trial. Expert Rev Neurother 9: 789-795, 2009. 